\title{
Continuation and maintenance treatments for persistent depressive disorder
}

Liebherz, Sarah ; Machmutow, Katja ; Jansen, Alessa ; Meister, Ramona ; Watzke, Birgit ; Härter, Martin Christian ; Kriston, Levente

\begin{abstract}
This is a protocol for a Cochrane Review (Intervention). The objectives are as follows: To assess the effects of pharmacological and psychological continuation and maintenance treatments for persistent depressive disorder, in comparison with each other; placebo (drug/attention placebo/non-specific treatment control); and treatment as usual (TAU). In addition, to assess the effects of combined psychological and pharmacological continuation and maintenance treatments, in comparison with either of these treatments alone.
\end{abstract}

DOI: https://doi.org/10.1002/14651858.cd012855

Posted at the Zurich Open Repository and Archive, University of Zurich ZORA URL: https://doi.org/10.5167/uzh-201389

Journal Article

Published Version

Originally published at:

Liebherz, Sarah; Machmutow, Katja; Jansen, Alessa; Meister, Ramona; Watzke, Birgit; Härter, Martin Christian; Kriston, Levente (2017). Continuation and maintenance treatments for persistent depressive disorder. Cochrane Library:CD012855..

DOI: https://doi.org/10.1002/14651858.cd012855 


\section{(E) Cochrane Library}

Cochrane Database of Systematic Reviews

\section{Continuation and maintenance treatments for persistent depressive disorder (Protocol)}

Liebherz S, Machmutow K, Jansen A, Meister R, Watzke B, Härter MC, Kriston L

www.cochranelibrary.com 


\section{TABLE OF CONTENTS}

HEADER . . . . . . . . . . . . . . . . . . . . . . . . . . . . . . . . . . . . . . . 1

ABSTRACT . . . . . . . . . . . . . . . . . . . . . . . . . . . . . . . . . . . . . . . 1

BACKGROUND . . . . . . . . . . . . . . . . . . . . . . . . . . . . . . . . . . 1

OBJECTIVES . . . . . . . . . . . . . . . . . . . . . . . . . . . . . . . . . . . . . .

METHODS . . . . . . . . . . . . . . . . . . . . . . . . . . . . . . . . . . . . . . 4

ACKNOWLEDGEMENTS . . . . . . . . . . . . . . . . . . . . . . . . . . . . . . . . . . . . . . . . .

REFERENCES . . . . . . . . . . . . . . . . . . . . . . . . . . . . . . . . . . . . . . 9

APPENDICES . . . . . . . . . . . . . . . . . . . . . . . . . . . . . . . . . . . . . . . . . . . . .

HISTORY . . . . . . . . . . . . . . . . . . . . . . . . . . . . . . . . . . . . . . . . 16

CONTRIBUTIONS OF AUTHORS . . . . . . . . . . . . . . . . . . . . . . . . . . . . . . . . . . . . . . . .

DECLARATIONS OF INTEREST . . . . . . . . . . . . . . . . . . . . . . . . . . . . . . . . . 17

SOURCES OF SUPPORT . . . . . . . . . . . . . . . . . . . . . . . . . . . . . . . . . . . . . . . . . . . . . . 
[Intervention Protocol]

\title{
Continuation and maintenance treatments for persistent depressive disorder
}

\author{
Sarah Liebherz ${ }^{1}$, Katja Machmutow ${ }^{2}$, Alessa Jansen ${ }^{1}$, Ramona Meister ${ }^{1}$, Birgit Watzke ${ }^{2}$, Martin Christian Härter ${ }^{1}$, Levente Kriston ${ }^{1}$ \\ ${ }^{1}$ Department of Medical Psychology, University Medical Center Hamburg-Eppendorf, Hamburg, Germany. ${ }^{2}$ Department of Clinical \\ Psychology and Psychotherapy, University of Zurich, Zurich, Switzerland
}

Contact address: Sarah Liebherz, Department of Medical Psychology, University Medical Center Hamburg-Eppendorf, Hamburg, Germany. s.liebherz@uke.de.

Editorial group: Cochrane Common Mental Disorders Group.

Publication status and date: New, published in Issue 11, 2017.

Citation: Liebherz S, Machmutow K, Jansen A, Meister R, Watzke B, Härter MC, Kriston L. Continuation and maintenance treatments for persistent depressive disorder. Cochrane Database of Systematic Reviews 2017, Issue 11. Art. No.: CD012855. DOI: 10.1002/14651858.CD012855.

Copyright (C) 2017 The Cochrane Collaboration. Published by John Wiley \& Sons, Ltd.

\begin{abstract}
A B S T R A C T
This is a protocol for a Cochrane Review (Intervention). The objectives are as follows:

To assess the effects of pharmacological and psychological continuation and maintenance treatments for persistent depressive disorder, in comparison with each other; placebo (drug/attention placebo/non-specific treatment control); and treatment as usual (TAU). In addition, to assess the effects of combined psychological and pharmacological continuation and maintenance treatments, in comparison with either of these treatments alone.
\end{abstract}

\section{B A C K G ROU N D}

\section{Description of the condition}

Persistent forms of depression that last for two years or longer represent a substantial proportion of depressive disorders (Boland 2002; Gilmer 2005; Keller 1992; Spijker 2002). Within the literature, four subtypes can be distinguished: (1) dysthymia, (2) chronic major depression, (3) recurrent major depression with incomplete remission between episodes, and (4) double depression (Dunner 2005). Dysthymic disorder is defined as a condition with mild depressive symptoms persisting for at least two years. Major depressive episode, chronic type, refers to a more severe condition that meets the full criteria for major depression continuously for a minimum of two years. Patients who have recovered to the point at which they no longer meet full criteria for a major depressive episode but continue to experience significant symptoms for at least two years are referred to as recurrent major depression with incomplete remission between episodes. The superimposition of a major depressive episode on antecedent dysthymia is referred to as double depression (Klein 2010). In the Diagnostic and Statistical Manual of Mental Disorders (DSM)-5 (APA 2013), the new diagnostic category of persistent depressive disorder was introduced subsuming dysthymic as well as chronic major depressive disorders.

The mean length of persistent depression is between 17 to 30 years (Gilmer 2005; Kocsis 2008), and the lifetime prevalence for persistent depressive disorders is estimated to range from $3 \%$ to $6 \%$ in current epidemiological studies from the US and Australia (Kessler 2005; Klein 2010; Murphy 2012). In comparison to acute forms of depression, persistent depressive disorders are associated 
with longer treatment duration; increased loss of physical wellbeing; increased comorbidity; more severe impairments in social, psychological, and emotional functioning; increased health care utilization; and more frequent suicide attempts and hospitalizations (Arnow 2003; Gilmer 2005). Thus, persistent depression is likely to make a large contribution to the high burden of disease that is associated with unipolar depression according to disabilityadjusted life years (DALYs) (WHO 2008).

\section{Description of the intervention}

Overall, a large number of different interventions exist for the treatment of unipolar depression, including psychological, pharmacological, and combined psychological and pharmacological therapies. Evidence from randomized controlled trials (RCTs), as well as meta-analyses, suggests that these interventions are effective in the acute treatment of depression, including persistent forms of depression (Cuijpers 2010; Cuijpers 2013; Imel 2008; Keller 2000; Kriston 2014; Spijker 2013; von Wolff 2012; von Wolff 2013). Still, there is also evidence that some patients do not respond to treatment, do not reach complete remission, and develop persisting residual symptoms in the long term (Epstein 2014). It is estimated that half of the people suffering from depressive disorders are developing a chronic course (Klein 2011).

Moreover, acute phase treatments often fail to prevent relapse (which is defined as the return of symptoms of depression before a full remission has been achieved) and recurrence (which is defined as the appearance of another new episode of depression after full remission of a previous episode has been achieved) in major depression. For example, after scheduled termination of acute phase cognitive therapy (CT), relapse or recurrence rates were found to be $29 \%$ in the first year and $54 \%$ in the second year (Vittengl 2007). In this same study, even when other depression-specific psychological therapies and even higher doses of pharmacotherapy were used after the acute-phase treatment, relapse and recurrence rates were still high (Vittengl 2007). One study has shown that $30 \%$ to $50 \%$ of patients considered to be remitted still have to deal with residual depressive symptoms (Nutt 2007).

Thus, following response to acute treatment, long-term continuation and maintenance therapy is required to protect patients from relapse or recurrence of symptoms. Continuation treatments are defined as treatments given to currently remitted patients (remission is defined as depressive symptoms dropping below case level) or to patients that previously responded to an antidepressant treatment. Maintenance therapy is given during recovery (which is defined as remission lasting longer than six months; Frank 1991; NICE 2010). The German National Disease Management Guideline (S3-Guideline) for Unipolar Depression recommends a combination of pharmacotherapy and psychological therapy as acute phase treatment for patients suffering from persistent forms of depression (DGPPN 2015). Additionally, a continued psychological therapy or pharmacotherapy, or both, is recommended to prevent relapse and recurrence. Specifically, the type of treatment that was successful in the acute phase is recommended to be continued (APA 2010; DGPPN 2015; NICE 2010).

However, these recommendations are based on a limited number of studies and expert opinion. Hence, a systematic search of evidence regarding the effectiveness of pharmacological, psychological, and combined pharmacological and psychological therapies as continuation and maintenance treatments for patients suffering from persistent forms of depression is needed.

\section{How the intervention might work}

Acute treatments aim to reduce depressive symptoms and re-establish psychosocial functioning. In comparison, continuation and maintenance treatments aim to maintain (or improve) the psychofunctional status reached by acute treatment, and to reduce the likelihood of relapse and recurrence in the long-term (DGPPN 2015). Therefore, continuation and maintenance treatments are considered to be more than a pure extension of acute treatments, because continuation/maintenance treatments differ in frequency and content over the course of the illness in comparison to acute treatments.

Psychological continuation and maintenance interventions are offered usually less frequently than acute psychological therapy, aiming to monitor symptoms and to integrate techniques and strategies into daily life in the long-term (DGPPN 2015). Different programmes that target the prevention of relapse and recurrence focus on a range of effect mechanisms. Cognitive therapy (CT) approaches focus on the generalization of skills achieved during acute therapy (Jarrett 1998), or on the cognitive content of negative thinking (Bockting 2005). Mindfulness-Based Cognitive Therapy (MBCT) was developed to reduce relapse and recurrence in depression (Piet 2011; Segal 2002), and teaches people to deal with negative feelings and thoughts as a part of their lives through becoming aware of negative cognitive patterns. Maintenance Interpersonal Psychotherapy (IPT) aims to complement skills gained in the acute phase therapy and teaches patients to take responsibility in the prevention of future episodes by recognizing and preventing stressing environmental and social circumstances (Beshai 2011). Still, it remains challenging to completely understand the mechanisms of preventing relapse and recurrence (Beshai 2011). The exact therapeutic mechanisms of antidepressants are still critically discussed (Pringle 2011). Most antidepressants seem to increase the concentrations of monoamine neurotransmitters (e.g. serotonin or noradrenaline) in the synaptic cleft (Berton 2006). However, the effect of most antidepressants fully develops after some weeks, indicating that neurophysiological changes of brain tissue (e.g. changes in sensitivity and frequency of receptors), occurring in the presence of a constant level of active ingredients, are necessary for permanent improvement. Depending on the type of active ingredient, antidepressants can have mood-enhancing, anxiolytic, or sedative effects and can increase or decrease inner drive. 
Moreover, the placebo effect is of particular importance in the treatment of depression. Several studies assume that the more severe the depressive symptoms are, the greater the benefit of antidepressants seem to be compared to placebo (Anderson 2008; Kirsch 2008). However, a recent meta-analysis performed on patient-level data regarding the response to antidepressant medication showed that initial depression severity and outcomes were similarly related in treatment and placebo groups (Rabinowitz 2016).

A number of studies have shown that the risk of relapse or recurrence of depression is associated with residual symptoms following acute treatment phases (APA 2010; NICE 2010). These findings have lead to the therapeutic goal of sustained remission and recommendations of international treatment guidelines to continue antidepressant medication after acute phase treatment (APA 2010; NICE 2010).

\section{Why it is important to do this review}

Research that focuses on the prevention of recurrence of depression was identified as a top priority in the recent project 'Depression: asking the right questions' (MQ 2016). The high prevalence and the severe personal, societal, and economic consequences of persistent depressive disorder (Gilmer 2005), underline the need for adequate treatment strategies. Growing evidence indicates that persistent depressive disorder responds well to several acute interventions, such as combined psychological and pharmacological treatments, although the number of RCTs is still limited (Spijker 2013). Yet, given the high rates of relapse and recurrences of depression following response to acute treatment, long-term continuation and maintenance therapy are of great importance (Beshai 2011).

Several RCTs have supported the effectiveness of continuation and maintenance therapies for depression has been supported (Browne 2002; Jarrett 2001; Jarrett 2013; Keller 2007; Klein 2004; Petersen 2010; Vittengl 2009). A meta-analysis on relapse prevention with antidepressant drug treatment of depressive disorders showed that continued antidepressant medication produced a robust reduction in relapse (Glue 2010). Another meta-analysis, Vittengl 2007, summarizes the findings of long-term effects of cognitive behavioural therapy (CBT). Patients who responded to acute treatment and continued to receive CBT showed a significant reduction in relapse and recurrence rates in comparison to inactive as well as active controls.

Although most evidence addresses acute treatments for persistent depressive disorder or long-term treatments for acute depressive episodes, some studies have been performed to address the effectiveness of long-term treatments of persistent depressive disorder (Gelenberg 2003; Harrison 1986; Keller 1998; Klein 2004; Kocsis 1996; Kocsis 2003; Koran 2001; Rouillon 1989; Stangier 2013). Until now, no systematic review on the comparative effectiveness of continuation and maintenance treatments for persistent depressive disorder has been available.
In summary, this systematic review may be highly relevant as:

- persistent depressive disorders have a high prevalence and serious personal, societal, and economic consequences;

- no evidence synthesis is available on continuation and maintenance treatments of persistent depressive disorders;

- high quality evidence synthesis is needed for clinical guideline recommendations.

\section{O B J E C T I VES}

To assess the effects of pharmacological and psychological continuation and maintenance treatments for persistent depressive disorder, in comparison with each other; placebo (drug/attention placebo/non-specific treatment control); and treatment as usual (TAU). In addition, to assess the effects of combined psychological and pharmacological continuation and maintenance treatments, in comparison with either of these treatments alone.

\section{METHODS}

\section{Criteria for considering studies for this review}

\section{Types of studies}

We will include RCTs, including cross-over and cluster-RCTs, and controlled clinical trials (CCTs). We have considered CCTs for inclusion as the number of RCTs may be limited. We will not apply any restrictions regarding other study design characteristics.

\section{Types of participants}

\section{Participant characteristics}

We will include participants 18 years of age and older of any gender and ethnicity.

\section{Diagnosis}

We will include participants who have a diagnosis of persistent depressive disorder or have had this diagnosis before their last previous acute treatment. The diagnosis of depression needs to rely on a formal classification system, such as the International Classification of Diseases (ICD) (WHO 1992), or DSM (APA 2013). Participants need to be either currently remitted from persistent depressive disorder or need to have at least partially responded to 
an acute intervention (at least $25 \%$ symptom reduction from baseline) at the beginning of the continuation or maintenance treatment. We will include participants described as "treatment resistant" if they fulfil the formerly mentioned criteria. As the distinction between subtypes of persistent depressive disorder (chronic major depression, dysthymia, double depression, or recurrent depression without a complete remission between episodes) is controversial, inclusion will be primarily driven by the duration of the existing depressive disorder. Consequently, we will include studies that investigate participants with chronic major depression, dysthymia, double depression, or recurrent depression without a complete remission between episodes if the target disorders are or have been of at least two years' duration. We will exclude studies that report to investigate "chronically depressed" participants without fulfilling these criteria (e.g. less than two years duration).

\section{Co-morbidities}

We will exclude studies that focus exclusively on persistently depressed participants with a specific concurrent mental or somatic disorder; we assume that the interventions in these types of studies (primarily) address the comorbid condition and are not focused exclusively on persistent depression. We will include studies that do not define specific concurrent mental or somatic conditions as inclusion criteria but report on comorbidities in addition to the persistent depressive disorder.

\section{Setting}

We will not place any restrictions based on setting.

\section{Subset data}

We will only consider studies in which both participants with persistent and acute forms of depression for inclusion if data are reported separately for the persistent subgroup (or if $80 \%$ or more of the total sample has a diagnosis of persistent depression). If randomization is based on the total sample, we will include studies and we will categorize them as non-RCTs.

\section{Types of interventions}

\section{Experimental Intervention}

We will consider pharmacological, psychological, and combined continuation and maintenance interventions for inclusion. Continuation treatments are defined as treatments given to currently remitted individuals or to individuals that previously responded to an antidepressant treatment, whereas maintenance treatments are defined as treatments given to people who are currently recovered. Continuation/maintenance treatments need to be started within one year after termination of an acute treatment. We will consider all interventions that satisfy these definitions. Additionally, we will consider for inclusion studies that do not report all the above mentioned criteria but report data on interventions that are clearly labelled as "continuation" or "maintenance" treatments. Pharmacological interventions include the oral administration of classified antidepressants:

- tricyclic antidepressants;

- selective serotonin reuptake inhibitors;

- monoamine oxidase inhibitors;

- alpha2-receptor-antagonists;

- selective noradrenalin dopamine reuptake inhibitors;

- melatonin receptor agonists; and

- serotonin 5 HT2C receptor antagonists;

as well as the following:

- non-classified antidepressants (Trazodone);

- lithium;

- Hypericum perforatum; and

- antipsychotic drugs;

as these drugs can be used (alone or in combination) in treating different forms of depression (DGPPN 2015).

Psychological therapies must fulfil the following criteria:

- the intervention must be based on a scientific theory (described in detail, manualized or referenced);

- at least one contact between therapist and participant either face-to-face or via telecommunication technologies (e.g. online therapy) must take place. Thus, for example, we will not consider the general dissemination of information material in form of leaflets in waiting rooms as a psychological therapy;

- the intervention must consider the personal needs of the participant or a group of participants and must be individually tailored in an interpersonal process. Thus, we will include group therapies.

Concerning psychological therapies, we will include behaviour therapy/behaviour modification, CBT, third wave CBTs, psychodynamic therapies, humanistic therapies, integrative therapies, systemic therapies, and other psychologically-oriented interventions (based on the definition of the Cochrane Common Mental Disorders Group).

Combined interventions include the administration of one or more pharmacological agents combined with one or more psychological therapy.

We will not consider for inclusion somatic (e.g. electroconvulsive therapy, vagus nerve stimulation, acupuncture), non-pharmacological (e.g. physical exercise, bright light therapy), and organizational (e.g. case management) interventions as including too many different interventions is likely to result in large clinical and methodological heterogeneity.

\section{Comparator intervention}


We will include both controlled and comparative effectiveness studies. The comparators may be:

- pharmacological placebo (participants receive placebo pills);

- attention-placebo/nonspecific control (participants receive a

treatment that involves nonspecific psychosocial factors);

- TAU;

- (other) psychological therapy;

- (other) pharmacological treatment;

- (other) combined psychological/pharmacological therapy.

\section{Types of outcome measures}

\section{Primary outcomes}

- Relapse or recurrence rate of depression, preferentially defined as:

(a) fulfilment of formal diagnostic criteria for depression (DSM, ICD); or

(b) exceeding a cut-off on a depression symptom rating scale used by the authors, specifically:

i. the Hamilton Depression Rating Scale (HDRS) (Hamilton 1960);

ii.the Montgomery -Åsberg Depression Rating Scale (MADRS)

(Montgomery 1979);

iii.the Beck-Depression-Inventory (BDI) (Beck 1996);

iv.the Inventory of Depressive Symptomatology (IDS) (Rush 2000);

v. the Patient Health Questionnaire (PHQ) (Spitzer 1999); or

vi.any other depression symptom scale.

Due to the long tradition of depression research, most instruments used in clinical trials are usually psychometrically sound. Such measures will be preferred throughout the review (either referenced and/or sufficient psychometric quality reported).

- Dropout due to any reason.

\section{Secondary outcomes}

- Symptom severity of depression at the end of treatment (metric outcome of depression scale as defined above);

- health-related quality of life e.g. WHO Quality of Life

(WHOQOL) (Skevington 2004);

- dropout due to adverse events;

- experiencing any adverse event;

- serious adverse events.

\section{Timing of outcome assessment}

The primary outcome time point will be the 'end of intervention' (regardless of the duration of the intervention). Additionally, we will evaluate outcomes at the time point 'one year after the end of intervention' provided sufficient data are available. If one-year data are unavailable, we will use data that range between six and 18 months after the end of intervention, with a preference for the time that was closest to one year after the end of intervention.

\section{Hierarchy of outcome measures}

If more than one diagnostic definition or depression symptom rating scale, or both, are available (concerning the outcome 'Relapse or recurrence rate of depression'), we will use the presented hierarchy to select measures (priority starting with (a) fulfilment of formal diagnostic criteria, continuing with (b) (i. HDRS), (ii. MADRS) etc.).

\section{Search methods for identification of studies}

\section{Electronic searches}

- The Cochrane Common Mental Disorders Group maintains a specialized register of RCTs: the CCMD-CTR (description in Appendix 1).

We will search the CCMD-CTR for study records using the following controlled search terms (condition only):

("chronic depression" or "dysthymia" or "dysthymic disorder" or "persistent depressive disorder" or "recurrent depression")

- We will search the CCMD-CTR for reference records using a more sensitive set of terms (condition only):

("chronic* depress"” or "double depress*" or dysthymi* or (depress* NEAR2 recurr*) or "persistent depressive disorder"): ti,ab,kw,ky,mh,mc,emt

[Key: ti=title; $\mathrm{ab}=\mathrm{abstract}$; $\mathrm{kw}=\mathrm{keyword} ; \mathrm{ky=additional} \mathrm{keywords;}$ $\mathrm{mh}=\mathrm{MeSH}$ terms; $\mathrm{mc}=\mathrm{MeSH}$ checkwords;emt=EMTREE terms] We will screen records for continuation and maintenance trials.

- As the review will include both RCTs and CCTs, we will conduct complementary searches on the following bibliographic databases using relevant subject headings and search syntax', appropriate to each resource:

○ OVID PsycINFO (search strategy listed in Appendix $1)$

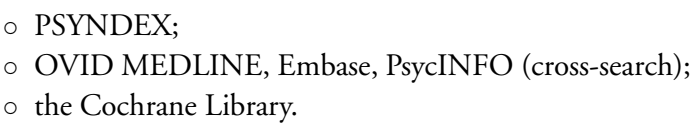

We will search databases from 1970 onwards (Embase from 1980); and we will not apply any other restriction on language or publication status to the searches.

We will screen records retrieved from the CCMD-CTR and PsycINFO searches prior to running other database searches. We will validate the search strategies to prevent the retrieval of too many irrelevant references (e.g. whether it is appropriate to include 
specific terms for 'maintenance' or 'continuation' of treatments for persistent depressive disorder).

\section{Searching other resources}

\section{Grey literature}

We will search the following sources of grey literature:

- ProQuest Dissertations \& Theses Database (

www.proquest.com/);

- Depression. The treatment and management of depression

in adults (NICE 2010);

- S3 Guideline/National Disease Management Guideline.

Unipolar Depression (DGPPN 2015);

- Canadian Network for Mood and Anxiety Treatments

(CANMAT). Clinical guidelines for the management of major

depressive disorder in adults (Kennedy 2009)

- Open Grey (www.opengrey.eu/).

\section{Handsearching}

As all relevant journals are included in the bibliographic databases being searched, we will not conduct any no further handsearches of journals.

\section{Reference lists}

We will check the reference lists of all included studies and relevant systematic reviews to identify additional studies missed from the original electronic searches (e.g. unpublished or in-press citations). Also we will conduct a cited reference search on the Web of Science.

\section{Correspondence}

We will contact the first author of each included study for information on unpublished or ongoing studies, or to request additional trial data.

\section{Data collection and analysis}

\section{Selection of studies}

Two review authors (KM, SL) will independently screen titles and abstracts for inclusion of all the potential studies identified as a result of the search and code them as either 'retrieve' (eligible or potentially eligible/unclear) or 'do not retrieve' (ineligible). We will retrieve the full-text study reports/publications and two review authors (KM, SL, or RM) will independently screen the full-texts and determine studies for inclusion. We will record the reasons for exclusion of the ineligible studies. We will resolve any disagreement through discussion or, if required, we will consult a fourth review author (AJ). We will identify and exclude duplicate records and will collate multiple reports that relate to the same study so that each study, rather than each report, is the unit of interest in the review. We will record the selection process in sufficient detail to complete a PRISMA flow diagram and a 'Characteristics of excluded studies' table.

\section{Data extraction and management}

We will use a data collection form, which has been piloted on at least one study in the review, to extract study characteristics and outcome data. Two review authors (KM, SL, or RM) will independently extract study characteristics and outcome data from included studies. We will extract the following study characteristics:

- methods: study design, time of randomization, total duration of study, location, study setting, and date of study (year);

- participants: number of participants $(\mathrm{N})$, mean age, age range, \% female, diagnostic subgroup, mean age of onset, length of current/last episode, number of previous episodes;

- interventions: intervention, comparison, type of acute treatment previous to continuation/maintenance treatment;

- outcomes: primary and secondary outcomes specified and collected, and time points reported;

- notes: funding of the trial.

We will note in the 'Characteristics of included studies' table if outcome data was not reported in a usable way. We will resolve disagreements by consensus or by involving a third review author (KM, SL, or RM). One review author (KM) will transfer data into Review Manager 5 (RevMan 5) (RevMan 2014). We will doublecheck that data is entered correctly by comparing the data presented in the systematic review with the study reports. A second review author (SL) will spot-check study characteristics for accuracy against the trial report.

\section{Main comparisons}

We have chosen seven main comparisons from the list of possible comparisons based on clinical importance and expected frequency of the comparisons in clinical trials:

- pharmacological continuation and maintenance therapies versus placebo;

- pharmacological continuation and maintenance therapies versus TAU;

- psychological continuation and maintenance therapies versus attention placebo/nonspecific control;

- psychological continuation and maintenance therapies versus TAU;

- psychological continuation and maintenance therapies versus pharmacological continuation and maintenance therapies; 
- combined psychological and pharmacological continuation and maintenance therapies versus pharmacological continuation and maintenance therapies alone;

- combined psychological and pharmacological continuation and maintenance therapies versus psychotherapeutic continuation and maintenance therapies alone.

\section{Assessment of risk of bias in included studies}

Two review authors (KM, SL, or RM) will independently assess the risk of bias for each included study using the criteria outlined in the Cochrane Handbook for Systematic Reviews of Interventions (Higgins 2011). We will resolve any disagreements by discussion or by involving another review author (KM, SL, or RM). We will assess the risk of bias according to the following domains:

- random sequence generation;

- allocation concealment;

- blinding of participants and personnel;

- blinding of outcome assessment;

- incomplete outcome data;

- selective outcome reporting;

- other bias.

We will judge each potential source of bias as either at high, low, or unclear risk of bias and will provide a supporting quotation from the study report together with a justification for our judgment in the 'Risk of bias' table. We will summarize the 'Risk of bias' judgments across different studies for each of the domains listed. Where information on risk of bias relates to unpublished data or correspondence with a trial author, we will note this in the 'Risk of bias' table.

We will use the ACROBAT-NRSI tool for assessing the quality of non-randomized studies in meta-analyses to assess the quality of non-RCTs (Sterne 2014). Moreover, we will assess recruitment bias, baseline imbalance, loss of clusters, incorrect analysis, and comparability with individually randomized trials in cluster-RCTs (Higgins 2011).

The risk of bias will be considered in sensitivity analyses. Moreover, we will take the risk of bias into account when we interpret the treatment effects.

\section{Measures of treatment effect}

\section{Dichotomous data}

In order to increase clinical applicability of the findings, we will calculate the relative risk of relapse/recurrence for the primary outcomes, as they are more likely to help clinicians to make informed decisions in specific clinical situations. If primary studies used time-to-event data, we will calculate pooled hazard ratios. For rare outcomes (adverse events, and possibly dropout rates) or endpoints with highly varying baseline rates, we will estimate the odds ratios.

\section{Continuous data}

We will analyse continuous data as mean differences (MD). If the included studies used different rating scales to assess the same outcome, we will calculate the standardized mean differences (SMD). We will enter data presented as a scale with a consistent direction of effect. We will undertake meta-analyses only where this is meaningful, i.e. if the treatments, participants, and the underlying clinical question are similar enough for useful pooling. We will narratively describe skewed data reported as medians and interquartile ranges if effect size calculation is not possible.

\section{Unit of analysis issues}

\section{Cluster-randomized trials}

As we expect a small number of overall available studies, we will include data from controlled clinical trials, cross-over trials, and cluster-randomized trials in the analysis, regardless of the level of randomization. Concerning cluster-randomized trials, we will include direct effect estimates of the primary studies, only if they were obtained from analyses that account for the clustering in the data (e.g. using a multilevel model). Otherwise, we will approximate the effect estimates using an inflated standard error that incorporates the design effect (Higgins 2011).

\section{Cross-over trials}

We will only use the first comparison (pre-crossover) meeting our inclusion criteria from cross-over trials.

\section{Studies with multiple treatment groups}

Concerning studies with multiple treatment groups, we will only consider data from the comparison of interest for each of the main objectives addressed in our review. If the study provides more than one comparison of interest for one of the main objectives, we will split the 'shared' group into two or more groups with smaller sample sizes to avoid including participants more than once in the analysis.

\section{Dealing with missing data}

In case of missing or unclear data, we will contact corresponding study authors or study sponsors in order to obtain key study characteristics and missing numerical outcome data when possible (e.g. when a study is identified as abstract only). We will document all requests and correspondences. 
For all studies, we will calculate effect sizes using the intention-totreat (ITT) principle, i.e. analysing all participants allocated to the respective study arm. For the primary outcome, we will include all randomized participants in the analyses irrespective of how the study authors defined their ITT sample. For all other outcomes we will follow the definition of the ITT sample provided by the study authors.

\section{Assessment of heterogeneity}

We will test statistical heterogeneity between study results for significance using Cochran's Q-test and will quantify it using the $\mathrm{I}^{2}$ statistic (Higgins 2003).

We will display results visually as forest plots. We expect considerable clinical heterogeneity between studies. We will consider the $\mathrm{I}^{2}$ statistic values as follows:

- $0 \%$ to $40 \%$ : might not be important;

- $30 \%$ to $60 \%$ : may represent moderate heterogeneity;

- $50 \%$ to $90 \%$ : may represent substantial heterogeneity; and

- $75 \%$ to $100 \%$ : considerable heterogeneity.

Based on this classification, we will consider $\mathrm{I}^{2}$ statistic values in the range of 50 to $100 \%$ as relevant statistical heterogeneity that is to be further explored. As "thresholds for the interpretation of $\mathrm{I}^{2}$ can be misleading, since the importance of inconsistency depends on several factors" (Higgins 2011), this is only a rough orientation. Therefore, we will decide on a case-by-case-basis if the determined heterogeneity needs to be further explored.

\section{Assessment of reporting biases}

We will test possible reporting biases and small-study effects using visual examination of funnel plots and applying Egger's test (Sterne 2001).

\section{Data synthesis}

We will perform all analyses by applying a random-effects model (DerSimonian 1986).

We plan to use random-effects models rather than fixed-effect ones, because we assume that the included studies will not be functionally equivalent and will show considerable clinical (concerning population, intervention) and methodological (concerning quality) heterogeneity. We will display results visually as forest plots. If it will not be possible to combine studies via meta-analysis, we will provide a narrative summary.

\section{Subgroup analysis and investigation of heterogeneity}

In order to identify possible treatment effect moderators, we will perform a priori defined subgroup analyses (in case of categorical predictors) or meta-regression analyses (in case of metric predictors) for the primary outcomes. We will test differences between subgroups formally (Bucher 1997; Deeks 2008; Song 2003). All meta-regression analyses will be performed using the restricted maximum likelihood estimate method, a recommended randomeffects approach that accounts for residual between-trial heterogeneity (Thompson 1999).

We will consider the following variables:

- subtype of persistent depressive disorder (dysthymia versus other): a possibly moderating effect of subtype would suggest that a distinction between these subtypes might be used for allocation of patients to treatments (differential indication). On the other hand, a possible homogeneity of effects across subtypes may suggest that a distinction is of little relevance in the day-to-day practice. We will test dysthymia against other subtypes as dysthymia is assumed to be the most frequently mentioned subtype;

- mean age of onset: the age of onset is known as a relevant predictor, it should be assessed if patients with early onset need different treatments;

- applied intervention (cognitive behavioral therapies versus other, selective serotonin reuptake inhibitors (SSRIs) versus other): as experience shows, cognitive behavioral approaches/ SSRIs are the most frequent forms of psychological therapies/ antidepressants to be studied. Therefore, we decided to test these approaches versus other approaches. Evidence on the best available treatments (in case of considerable differences) is indispensable for guideline recommendations;

\section{- duration of continuation/maintenance treatment}

(weeks): for guideline recommendations and clinical practice, it is indispensable to know if different treatment durations result in different outcomes, e.g. if longer treatments lead to better outcomes.

In case of considerable heterogeneity between study results that we cannot explain by the a priori defined subgroup and meta-regression analyses, we will perform a series of a posteriori (explorative) meta-regression analyses to identify sources of heterogeneity. We will clearly label a priori and a posteriori analyses as such.

\section{Sensitivity analysis}

We will perform sensitivity analyses by excluding studies with a high or unclear risk of bias (separately for each of the seven domains according to the Cochrane 'Risk of bias' tool) or outlying findings, or both. We will compare results to those acquired with data from all studies in order to control for possible effects of study quality on pooled effects.

We will perform the following additional sensitivity analyses:

- excluding trials without a randomization on person level (second phases of cross-over trials, CCTs, and clusterrandomized trials); and

- excluding trials without (re-)randomization immediately before the continuation/maintenance phase in order to control for possible design effects. 


\section{'Summary of findings' table}

We will construct 'Summary of findings' tables for the seven main comparisons: effectiveness of pharmacotherapy and effectiveness of psychological therapy for persistent depressive disorder, the effectiveness of psychological therapy in comparison to pharmacotherapy for persistent depressive disorder, and the effectiveness of combined treatments in comparison to pure pharmacological/ pure psychological therapies for persistent depressive disorder. The 'Summary of findings' tables will include a summary of the quality of evidence, the magnitude of effects of the according intervention, and a summary of available data on main outcomes. We will express findings as measures of risk ratio and absolute risk for the main outcomes of relapse/recurrence and dropout. We will use the GRADE approach to assess the quality of the evidence (Guyatt 2011).

\section{ACKNOWLEDGEMENTS}

The project COMACHRON is funded by the German Federal Ministry of Education and Research (Bundesministerium für Bildung und Forschung (BMBF); funding code 01KG1403). The $\mathrm{BMBF}$ grants the support of the project on the basis of the project application, but the BMBF has no influence on the study design; the data collection, analysis, and interpretation; the writing of the manuscript; or the decision to submit the paper for publication.

\section{CRG funding acknowledgement}

The National Institute for Health Research (NIHR) is the largest single funder of the Cochrane Common Mental Disorders Group.

\section{Disclaimer}

The views and opinions expressed therein are those of the protocol authors and do not necessarily reflect those of the NIHR, the NHS, or the Department of Health.

\section{RE F E R E N C E S}

\section{Additional references}

\section{Anderson 2008}

Anderson IM, Ferrier IN, Baldwin RC, Cowen PJ, Howard

L, Lewis G, et al. Evidence-based guidelines for treating depressive disorders with antidepressants: a revision of the 2000 British Association for Psychopharmacology guidelines. Journal of Psychopharmacology (Oxford, England) 2008;22(4):343-96.

\section{APA 2010}

American Psychiatric Association (APA). Practice Guedlineline for the Treatment of Patients with Major Depressive Disorder. 3rd Edition. Washington, DC: American Psychiatric Association, 2010.

\section{APA 2013}

American Psychiatric Association (APA). Diagnostic and Statistical Manual of Mental Disorders (DSM-5). 5th Edition. Washington, DC: American Psychiatric Association, 2013.

\section{Arnow 2003}

Arnow BA, Constantino MJ. Effectiveness of psychotherapy and combination treatment for chronic depression. Journal of Clinical Psychology 2003;59(8):893-905.

\section{Beck 1996}

Beck AT, Steer RA, Brown GK. Manual for the Beck Depression Inventory-II. San Antonio, Texas: Psychological Corporation, 1996.

\section{Berton 2006}

Berton O, Nestler EJ. New approaches to antidepressant drug discovery: beyond monoamines. Nature Reviews. Neuroscience 2006;7(2):137-51.
Beshai 2011

Beshai S, Dobson KS, Bockting CL, Quigley L. Relapse and recurrence prevention in depression: current research and future prospects. Clinical Psychology Review 2011;31(8): 1349-60.

\section{Bockting 2005}

Bockting CL, Schene AH, Spinhoven P, Koeter MW, Wouters LF, Huyser J, et al. Preventing relapse/recurrence in recurrent depression with cognitive therapy: a randomized controlled trial. Journal of Consulting and Clinical Psychology 2005;73(4):647-57.

\section{Boland 2002}

Boland RJ, Keller MB. Course and outcome of depression. In: Gotlib IH, Hammen CL editor(s). Handbook of Depression. New York: Guilford Press, 2002:43-60.

\section{Browne 2002}

Browne G, Steiner M, Roberts J, Gafni A, Byrne C, Dunn E, et al. Sertraline and/or interpersonal psychotherapy for patients with dysthymic disorder in primary care: 6month comparison with longitudinal 2-year follow-up of effectiveness and costs. Journal of Affective Disorders 2002; 68(2-3):317-30.

\section{Bucher 1997}

Bucher HC, Guyatt GH, Griffith LE, Walter SD. The results of direct and indirect treatment comparisons in meta-analysis of randomized controlled trials. Journal of Clinical Epidemiology 1997;50(6):683-91.

\section{Cuijpers 2010}

Cuijpers P, van Straten A, Schuurmans J, van OP, Hollon SD, Andersson G. Psychotherapy for chronic major 
depression and dysthymia: a meta-analysis. Clinical Psychology Review 2010;30(1):51-62.

\section{Cuijpers 2013}

Cuijpers P, Sijbrandij M, Koole SL, Andersson G, Beekman AT, Reynolds CF. The efficacy of psychotherapy and pharmacotherapy in treating depressive and anxiety disorders: a meta-analysis of direct comparisons. World Psychiatry 2013;12(2):137-48.

\section{Deeks 2008}

Deeks JJ, Altman DG, Bradburn MJ. Chapter 15: Statistical methods for examining heterogeneity and combining results from several studies in meta-analysis. In: Egger M, Smith GD, Altman DG editor(s). Systematic Reviews in Health Care: Meta-Analysis in Context. 2nd Edition. London: BMJ Publishing Group, 2008:285-312.

\section{DerSimonian 1986}

DerSimonian R, Laird N. Meta-analysis in clinical trials. Controlled Clinical Trials 1986;7(3):177-88.

\section{DGPPN 2015}

DGPPN, BÄK, KBV, AWMF, AkdÄ, BPtK, BApK, DAGSHG, DEGAM, DGPM, DGPs, DGRW (Editors) for the Guideline Group Unipolar Depression. S3-Guideline/ National Disease Management Guideline Unipolar Depression - Long Version, 1st edition: Version 5. Available from www.leitlinien.de/mdb/downloads/nvl/depression/ depression-1 aufl-vers5-lang.pdf (accessed 12 August 2015).

\section{Dunner 2005}

Dunner DL. Dysthymia and double depression.

International Review of Psychiatry 2005;17(1):3-8.

\section{Epstein 2014}

Epstein I, Szpindel I, Katzman MA. Pharmacological approaches to manage persistent symptoms of major depressive disorder: rationale and therapeutic strategies. Psychiatry Research 2014;220 Suppl 1:S15-33.

\section{Frank 1991}

Frank E, Prien RF, Jarrett RB, Keller MB, Kupfer DJ, Lavori PW, et al. Conceptualization and rationale for consensus definitions of terms in major depressive disorder. Remission, recovery, relapse, and recurrence. Archives of General Psychiatry 1991;48(9):851-5.

\section{Gelenberg 2003}

Gelenberg AJ, Trivedi MH, Rush AJ, Thase ME, Howland $\mathrm{R}$, Klein DN, et al. Randomized, placebo-controlled trial of nefazodone maintenance treatment in preventing recurrence in chronic depression. Biological Psychiatry 2003;54(8): 806-17.

Gilmer 2005

Gilmer WS, Trivedi MH, Rush AJ, Wisniewski SR, Luther J, Howland RH, et al. Factors associated with chronic depressive episodes: a preliminary report from the STARD project. Acta Psychiatrica Scandinavica 2005;112(6): 425-33.

\section{Glue 2010}

Glue P, Donovan MR, Kolluri S, Emir B. Meta-analysis of relapse prevention antidepressant trials in depressive disorders. Australian and New Zealand Journal of Psychiatry 2010;44(8):697-705.

\section{Guyatt 2011}

Guyatt G, Oxman AD, Akl EA, Kunz R, Vist G, Brozek J, et al. GRADE guidelines: 1. Introduction-GRADE evidence profiles and summary of findings tables. Journal of Clinical Epidemiology 2011;64(4):383-94.

\section{Hamilton 1960}

Hamilton A. A rating scale for depression. Journal of Neurology, Neurosurgery and Psychiatry 1960;23:56-62.

\section{Harrison 1986}

Harrison W, Rabkin J, Stewart JW, McGrath PJ, Tricamo E, Quitkin F. Phenelzine for chronic depression: a study of continuation treatment. Journal of Clinical Psychiatry 1986; 47(7):346-9.

\section{Higgins 2003}

Higgins JP, Thompson SG, Deeks JJ, Altman DG. Measuring inconsistency in meta-analyses. BMJ 2003;327 (7414):557-60

Higgins 2011

Higgins JP, Green S, editor(s). Cochrane Handbook for Systematic Reviews of Interventions Version 5.1.0 (updated March 2011). The Cochrane Collaboration, 2011. Available from handbook.cochrane.org.

Imel 2008

Imel ZE, Malterer MB, McKay KM, Wampold BE. A meta-analysis of psychotherapy and medication in unipolar depression and dysthymia. Journal of Affective Disorders 2008;110(3):197-206.

\section{Jarrett 1998}

Jarrett RB, Basco MR, Risser R, Ramanan J, Marwill $\mathrm{M}$, Kraft $\mathrm{D}$, et al. Is there a role for continuation phase cognitive therapy for depressed outpatients?. Journal of Consulting and Clinical Psychology 1998;66(6):1036-40.

\section{Jarrett 2001}

Jarrett RB, Kraft D, Doyle J, Foster BM, Eaves GG, Silver PC. Preventing recurrent depression using cognitive therapy with and without a continuation phase: a randomized clinical trial. Archives of General Psychiatry 2001;58(4): 381-8.

\section{Jarrett 2013}

Jarrett RB, Minhajuddin A, Gershenfeld H, Friedman ES Thase ME. Preventing depressive relapse and recurrence in higher-risk cognitive therapy responders: a randomized trial of continuation phase cognitive therapy, fluoxetine, or matched pill placebo. JAMA Psychiatry 2013;70(11): 1152-60.

\section{Keller 1992}

Keller MB, Lavori PW, Mueller TI, Endicott J, Coryell W, Hirschfeld RM, et al. Time to recovery, chronicity, and levels of psychopathology in major depression. A 5-year prospective follow-up of 431 subjects. Archives of General Psychiatry 1992;49(10):809-16.

\section{Keller 1998}

Keller MB, Gelenberg AJ, Hirschfeld RM, Rush AJ, Thase $\mathrm{ME}$, Kocsis JH, et al. The treatment of chronic depression, 
Part 2: A double-blind, randomized trial of sertraline and imipramine. Journal of Clinical Psychiatry 1998;59(11): 598-607.

\section{Keller 2000}

Keller MB, McCullough JP, Klein DN, Arnow B, Dunner DL, Gelenberg AJ, et al. A comparison of nefazodone, the cognitive behavioral-analysis system of psychotherapy, and their combination for the treatment of chronic depression. New England Journal of Medicine 2000;342(20):1462-70.

\section{Keller 2007}

Keller MB, Trivedi MH, Thase ME, Shelton RC, Kornstein SG, Nemeroff CB, et al. The Prevention of Recurrent Episodes of Depression with Venlafaxine for Two Years (PREVENT) Study: Outcomes from the 2-year and combined maintenance phases. Journal of Clinical Psychiatry 2007;68(8):1246-56.

\section{Kennedy 2009}

Kennedy SH, Lam RW, Parikh SV, Patten SB, Ravindran AV. Canadian Network for Mood and Anxiety Treatments (CANMAT) clinical guidelines for the management of major depressive disorder in adults. Journal of Affective Disorders 2009;117(Suppl 1):S1-2.

\section{Kessler 2005}

Kessler RC, Berglund P, Demler O, Jin R, Merikangas $\mathrm{KR}$, Walters EE. Lifetime prevalence and age-of-onset distributions of DSM-IV disorders in the National Comorbidity Survey Replication. Archives of General Psychiatry 2005;62(6):593-602.

\section{Kirsch 2008}

Kirsch I, Deacon BJ, Huedo-Medina TB, Scoboria A, Moore TJ, Johnson BT. Initial severity and antidepressant benefits: a meta-analysis of data submitted to the Food and Drug Administration. PLoS Medicine 2008;5(2):e45.

\section{Klein 2004}

Klein DN, Santiago NJ, Vivian D, Blalock JA, Kocsis JH, Markowitz JC, et al. Cognitive-behavioral analysis system of psychotherapy as a maintenance treatment for chronic depression. Journal of Consulting and Clinical Psychology 2004;72(4):681-8.

Klein 2010

Klein DN. Chronic depression: diagnosis and classification. Current Directions in Psychological Science 2010;19(2): $96-100$.

Klein 2011

Klein JP, Steinlechner S, Sipos V, Schweiger U. Psychotherapie chronischer Depression nach dem Cognitive Behavioral Analysis System of Psychotherapy (CBASP)Konzept. Psychotherapie, Psychosomatik, Medizinische Psychologie 2011;61(12):526-35.

\section{Kocsis 1996}

Kocsis JH, Friedman RA, Markowitz JC, Leon AC, Miller NL, Gniwesch L, et al. Maintenance therapy for chronic depression. A controlled clinical trial of desipramine. Archives of General Psychiatry 1996;53(9):769-74.

\section{Kocsis 2003}

Kocsis JH, Rush AJ, Markowitz JC, Borian FE, Dunner DL, Koran LM, et al. Continuation treatment of chronic depression: a comparison of nefazodone, cognitive behavioral analysis system of psychotherapy, and their combination. Psychopharmacology Bulletin 2003;37(4): $73-87$.

\section{Kocsis 2008}

Kocsis JH, Gelenberg AJ, Rothbaum B, Klein DN, Trivedi $\mathrm{MH}$, Manber R, et al. Chronic forms of major depression are still undertreated in the 21st century: systematic assessment of 801 patients presenting for treatment. Journal of Affective Disorders 2008;110(1-2):55-61.

\section{Koran 2001}

Koran LM, Gelenberg AJ, Kornstein SG, Howland RH, Friedman RA, DeBattista C, et al. Sertraline versus imipramine to prevent relapse in chronic depression. Journal of Affective Disorders 2001;65(1):27-36.

\section{Kriston 2014}

Kriston L, von Wolff A, Westphal A, Hölzel LP, Härter M. Efficacy and acceptability of acute treatments for persistent depressive disorder: a network meta-analysis. Depression and Anxiety 2014;31(8):621-30.

\section{Montgomery 1979}

Montgomery SA, Asberg M. A new depression scale designed to be sensitive to change. British Journal of Psychiatry: the Journal of Mental Science 1979;134:382-9.

\section{MQ 2016}

MQ: Transforming mental health through research. Depression: asking the right questions. Project report. Identifying priorities for depression research. https:// www.mqmentalhealth.org/articles/depression-prioritysetting-partnership (accessed 22 October 2017).

Murphy 2012

Murphy JA, Byrne GJ. Prevalence and correlates of the proposed DSM- 5 diagnosis of Chronic Depressive Disorder. Journal of Affective Disorders 2012;139(2):172-80.

\section{NICE 2010}

National Institute for Health \& Clinical Excellence. Depression. The treatment and management of depression in adults (updated edition): National Clinical Practice Guideline 90. Leicester and London: British Psychological Society and the Royal College of Psychiatrists, 2010.

Nutt 2007

Nutt D, Demyttenaere K, Janka Z, Aarre T, Bourin M, Canonico PL, et al. The other face of depression, reduced positive affect: the role of catecholamines in causation and cure. Journal of Psychopharmacology (Oxford, England) 2007; 21(5):461-71.

\section{Petersen 2010}

Petersen TJ, Pava JA, Buchin J, Matthews JD, Papakostas GI, Nierenberg AA, et al. The role of cognitive-behavioral therapy and fluoxetine in prevention of recurrence of major depressive disorder. Cognitive Therapy and Research 2010;34 (1):13-23. 
Piet 2011

Piet J, Hougaard E. The effect of mindfulness-based cognitive therapy for prevention of relapse in recurrent major depressive disorder: a systematic review and metaanalysis. Clinical Psychology Review 2011;31(6):1032-40.

Pringle 2011

Pringle A, Browning M, Cowen PJ, Harmer CJ. A cognitive neuropsychological model of antidepressant drug action. Progress in Neuro-psychopharmacology \& Biological Psychiatry 2011;35(7):1586-92.

Rabinowitz 2016

Rabinowitz J, Werbeloff N, Mandel FS, Menard F, Marangell L, Kapur S. Initial depression severity and response to antidepressants v. placebo: patient-level data analysis from 34 randomised controlled trials. British Journal of Psychiatry: the Journal of Mental Science 2016;209 (5):427-8.

RevMan 2014 [Computer program]

Nordic Cochrane Centre, The Cochrane Collaboration. Review Manager 5 (RevMan 5). Version 5.3. Copenhagen: Nordic Cochrane Centre, The Cochrane Collaboration, 2014.

Rouillon 1989

Rouillon F, Phillips R, Serrurier D, Ansart E, Gérard MJ. Prophylactic efficacy of maprotiline on relapses of unipolar depression [Rechutes de dépression unipolaire et efficacité de la maprotiline]. L'Encéphale: Revue de psychiatrie clinique biologique et thérapentique 1989;15(6):527-34.

\section{Rush 2000}

Rush AJ, Carmody T, Reimitz P-E. The Inventory of Depressive Symptomatology (IDS): Clinician (IDS-C) and Self-Report (IDS-SR) ratings of depressive symptoms. International Journal of Methods in Psychiatric Research 2000; 9(2):45-59.

\section{Segal 2002}

Segal ZV, Williams JMG, Teasdale JD. Mindfulness-based Cognitive Therapy for Depression: a New Approach to Preventing Relapse. New York: Guilford, 2002.

\section{Skevington 2004}

Skevington SM, Lotfy M, O'Connell KA, WHOQOL Group. The World Health Organization's WHOQOLBREF quality of life assessment: psychometric properties and results of the international field trial. A report from the WHOQOL group. Quality of Life Research: an international journal of quality of life aspects of treatment, care and rehabilitation 2004;13(2):299-310.

\section{Song 2003}

Song F, Altman DG, Glenny A-M, Deeks JJ. Validity of indirect comparison for estimating efficacy of competing interventions: empirical evidence from published metaanalyses. BMJ (Clinical research ed.) 2003;326(7387):472.

\section{Spijker 2002}

Spijker J, de Graaf R, Bijl RV, Beekman AT, Ormel J, Nolen WA. Duration of major depressive episodes in the general population: results from The Netherlands Mental Health
Survey and Incidence Study (NEMESIS). British Journal of Psychiatry: the Journal of Mental Science 2002;181:208-13.

\section{Spijker 2013}

Spijker J, van Straten A, Bockting C L, Meeuwissen JAC, van Balkom A. Psychotherapy, antidepressants, and their combination for chronic major depressive disorder: a systematic review. Canadian Journal of Psychiatry 2013;58 (7):386-92.

\section{Spitzer 1999}

Spitzer RL, Kroenke K, Williams JB. Validation and utility of a self-report version of PRIME-MD: the PHQ primary care study. Primary Care Evaluation of Mental Disorders. Patient Health Questionnaire. JAMA 1999;282(18): $1737-44$.

\section{Stangier 2013}

Stangier U, Hilling C, Heidenreich T, Risch AK, Barocka A, Schlosser R, et al. Maintenance cognitive-behavioral therapy and manualized psychoeducation in the treatment of recurrent depression: a multicenter prospective randomized controlled trial. American Journal of Psychiatry 2013;170(6): 624-32.

\section{Sterne 2001}

Sterne JAC, Egger M, Smith GD. Investigating and dealing with publication and other biases in meta-analysis. $B M J$ 2001;323(7304):101-5.

\section{Sterne 2014}

Sterne JAC, Higgins JPT, Reeves BC, on behalf of the development group for ACROBAT-NRSI. A Cochrane Risk Of Bias Assessment Tool: for Non-Randomized Studies of Interventions (ACROBAT-NRSI), Version 1.0.0. Available from http://www.riskofbias.info (accessed 24 June 2015).

\section{Thompson 1999}

Thompson SG, Sharp SJ. Explaining heterogeneity in metaanalysis: a comparison of methods. Statistics in Medicine 1999; 18(20):2693-708.

\section{Vittengl 2007}

Vittengl JR, Clark LA, Dunn TW, Jarrett RB. Reducing relapse and recurrence in unipolar depression: a comparative meta-analysis of cognitive-behavioral therapy's effects. Journal of Consulting and Clinical Psychology 2007;75(3): 475-88.

\section{Vittengl 2009}

Vittengl JR, Clark LA, Jarrett RB. Continuation-phase cognitive therapy's effects on remission and recovery from depression. Journal of Consulting and Clinical Psychology 2009;77(2):367-71.

\section{von Wolff 2012}

von Wolff A, Hölzel LP, Westphal A, Härter M, Kriston L. Combination of pharmacotherapy and psychotherapy in the treatment of chronic depression: a systematic review and meta-analysis. BMC Psychiatry 2012;12:61.

von Wolff 2013 von Wolff A, Hölzel LP, Westphal A, Härter M, Kriston L. Selective serotonin reuptake inhibitors and tricyclic antidepressants in the acute treatment of chronic depression 
and dysthymia: a systematic review and meta-analysis. Journal of Affective Disorders 2013;144(1-2):7-15.

\section{WHO 1992}

World Health Organization. International Classification of Mental and Behavioural Disorders, 10th Revision (ICD-10).

Geneva: World Health Organization, 1992.

\section{WHO 2008}

World Health Organization. The Global Burden of Disease 2004 update. Geneva: World Health Organization, 2008.

\section{References to other published versions of this review}

von Wolff 2014

von Wolff A, Liebherz S. Comparative effectiveness of Continuation and Maintenance Treatments for Chronic Depression (COMACHRON): a systematic review. PROSPERO 2014:CRD42014009928. Available from www.crd.york.ac.uk/PROSPERO/display_record.asp?ID= CRD42014009928 (accessed 12 August 2014).

* Indicates the major publication for the study

\section{A P P E N D I C E S}

\section{Appendix I. Search strategy}

\section{Cochrane Common Mental Disorders Specialized Register (CCMD-CTR)}

The Cochrane Common Mental Disorders Group maintains a specialized register of RCTs: the CCMD-CTR. This register contains over 39,000 reference records (reports of RCTs) for depression, anxiety, and other common mental disorders. A percentage of the reference records have been tagged to 12,500 individual, PICO-coded (PICO = participants, interventions, comparators, outcomes) study records (with coding based on the EU-Psi coding manual). Reports of trials for inclusion in the register are collated from (weekly) generic searches of MEDLINE, Embase, and PsycINFO; quarterly searches of the Cochrane Central Register of Controlled Trials (CENTRAL); and review-specific searches of additional databases. Reports of trials are also sourced from international trial registries, drug companies, the handsearching of key journals, conference proceedings, and other (non-Cochrane) systematic reviews and metaanalyses. Details of CCMD's core search strategies can be found on the Group's website.

\section{OVID PsycINFO}

[Condition]

1. (chronic* $^{*}$ depress*).ti,ab,id.

2. (double depress $\left.{ }^{*}\right)$.ti,ab,id.

3. DYSTHYMIC DISORDER/

4. MAJOR DEPRESSION/ and ("CHRONICITY (Disorders)"/ or CHRONIC ILLNESS/)

5. (dysthymi*).ti,ab,id.

6. RECURRENT DEPRESSION/

7. (depress* adj2 recurr*).ti,ab,id.

8. persistent depressive disorder.ti,ab,id.

9. or/1-8

[Maintenance]

10. MAINTENANCE THERAPY/

11. (maintenance or maintained).ti,ab,id.

12. continuation.ti,ab,id.

13. (stable or stabilise*1).ab.

14. RELAPSE PREVENTION/

15. "RELAPSE (Disorders)"।

16. or/10-15

[Controlled Trials Filter]

17. exp EXPERIMENTAL DESIGN/

Continuation and maintenance treatments for persistent depressive disorder (Protocol)

Copyright () 2017 The Cochrane Collaboration. Published by John Wiley \& Sons, Ltd. 


\section{TREATMENT EFFECTIVENESS EVALUATION/ \\ 19. MENTAL HEALTH PROGRAM EVALUATION/}

20. (empirical study or longitudinal study or prospective study or quantitative study).md.

21. "2000".md. [treatment outcome/clinical study]

22. RETROSPECTIVE STUDIES/

23. EVIDENCE BASED PRACTICE/

24. (study or trial or treatment* or intervention or therap* or psychotherap*).ti.

25. (control* adj3 (group*1 or study or trial)).ti,ab,id.

26. (waitlist* ${ }^{*}$ wait list* or treatment* as usual or TAU or care as usual or standard care or standard treatment*).ti,ab,id.

27. placebo.ti,ab,id.

28. PLACEBO/

29. (RCT or random*).ti,ab,id.

30. (crossover* or cross over*).ti,ab,id.

31. (quasi experimental).ti,ab,id.

32. (longitudinal or cohort).ti,ab,id.

33. (case adj (control or report or series)).ti,ab,id.

34. (cross-sectional).ti,ab,id.

35. (experimental or quantitative or pilot).ti,ab,id.

36. or/17-35

37. (9 and 16 and 36)

[Psychotherapies]

38. exp PSYCHOTHERAPY/

39. exp PSYCHOTHERAPEUTIC TECHNIQUES/

40. exp COGNITIVE TECHNIQUES/

41. $\exp$ COUNSELING/

42. 3300.cc. [Classification Code: Health \& Mental Health Treatment \& Prevention]

43. 3310.cc. [Classification Code: Psychotherapy \& Psychotherapeutic Counseling]

44. 3311.cc. [Classification Code: Cognitive Therapies]

45. 3312.cc. [Classification Code: Behavior Therapy \& Behavior Modification]

46. 3313.cc. [Classification Code: Group \& Family Therapy]

47. 3314.cc. [Classification Code: Interpersonal \& Client Centered \& Humanistic Therapy]

48. 3315.cc. [Classification Code: Psychoanalytic Therapy]

49. (CBT or c-CBT or iCBT or coping skills or counsel?ing or mindfulness or psychoanal* or psychotherap* or rehabilitat*).ti,ab,id.

50. ((psychologic* or psychodynamic or behavio?r or cognitive) adj3 (intervent* ${ }^{*}$ or therap* or treat* or manag*)).ti,ab,id.

51. (Abreaction or Acting Out or Adlerian or Adolescent Psychotherap* or Age Regression or Analytical Psychotherap* or Anger Control or Anger Management or Art Therap* or Assertive* Training or Autogenic Training or Autosuggestion or Aversion Therap* or Balint Group or Behavio?r Contracting or Behavio?r Modification or Behavio?r Therap* or Bibliotherap* or Biofeedback or Body Psychotherap* or Brief Psychotherap* or Caregiver Support or Child Psychotherap* or Client Cent* Therapy or Cognitive Behavio?r Therap* or Cognitive Behavio? ral Stress Management or Cognitive Rehabilitation or Cognitive Restructuring or Cognitive Therap* or Colo?r Therap* or Conjoint Therap* or Contingency Management or Conversion Therap* or Conversational Therap* or Countertransference or Couples Therap* or Covert Sensitization or Crisis Intervention).ti,ab,id,de.

52. (Dance Therap* or Dialectical Behavio?r Therap* or (Dream* adj3 Analys*) or Eclectic Psychotherap* or Eclectic Therap* or Emotion* Focus* Therap* or Emotional Freedom Technique or Encounter Group Therap* or Existential Therap* or Experiential Psychotherap* or Exposure Therap* or Expressive Psychotherap* or Eye Movement Desensiti\#ation or Family Therap* or Free Association or Geriatric Psychotherap* or Gestalt Therap* or Griefwork or Group Psychotherap* or Group Therap* or Guided Image* or Holistic Psychotherap* or Humanistic Psychotherap* or Hypnosis or Hypnotherapy or Hypnotiłzability or Implosive Therap* or Individual Psychotherap* or Insight Therap* or Integrative Psychotherap* or Integrative Therap* or Interpersonal Psychotherap*).ti,ab,id,de.

53. (Logotherap* or Marathon Group Therap* or Marital Therap* or Meditation or Mental Healing or Metacognitive Therap* or Milieu Therap* or Mind train* or Morita Therap* or Music Therap* or Narrative Therap* or Nondirective Therap* or Personal Construct Therap* or Person Cent* Therap* or Persuasion Therap* or Pet Therap* or Play Therap* or Primal Therap* or Problem Solving Therap* or Psychoanalysis or Psychoanalytic Therap* or Psychodrama or Psychodynamic Psychotherapy or Psychotherapeutic Counsel* $^{*}$ or Psychotherapeutic Processes or Psychotherapeutic Training or (Psychotherap* adj3 Rational-Emotive)) .ti,ab,id,de.

Continuation and maintenance treatments for persistent depressive disorder (Protocol)

Copyright ( 2017 The Cochrane Collaboration. Published by John Wiley \& Sons, Ltd. 
54. (Rational Emotive Behavio?r Therap* or Reality Therap* or Reciprocal Inhibition Therap* or Relationship Therap* or Relaxation Stress Management or Relaxation Technique* or Relaxation Therap* or Relaxation Training or Reminiscence Therap* or Role Playing or Self Analys* or Self Esteem Building or Sensitivity Training Group* or Sex Therap* or Sleep Phase Chronotherap* or Socioenvironmental Therap* or Sociotherap* or Solution Focused Therap* or Support Group* or (Support adj3 Psycho*) or Systematic Desensiti\#ation or Therapeutic Communit* or Transactional Analysis or Validation Therap*).ti,ab,id,de.

55. or/38-54

[Antidepressants]

56. PSYCHOPHARMACOLOGY/ or NEUROPSYCHOPHARMACOLOGY/

57. 3340.cc. [Classification Code: Clinical Psychopharmacology]

58. $\exp$ ANTIDEPRESSANT DRUGS/

59. NEUROTRANSMITTER UPTAKE INHIBITORS/ or exp SEROTONIN NOREPINEPHERINE REUPTAKE INHIBITORS/ or exp SEROTONIN REUPTAKE INHIBITORS/

60. exp MONOAMINE OXIDASE INHIBITORS/

61. exp TRICYCLIC ANTIDEPRESSANT DRUGS/

62. (antidepress* or anti depress* or MAOI* or monoamine oxidase inhibit* or ((serotonin or norepinephrine or noradrenaline or nor epinephrine or nor adrenaline or neurotransmitt* or dopamine*) and (uptake or reuptake or re-uptake)) or noradrenerg* or antiadrenergic or anti adrenergic or SSRI* or SNRI* or TCA $^{*}$ or tricyclic* or tetracyclic* or heterocyclic*).ti,ab,id,de.

63. (Agomelatine or Alnespirone or Amoxapine or Amfebutamone or Amiflamine or Amineptine or Amitriptylin* or Amitriptylinoxide or Amoxapine or (Atomoxetine or Tomoxetine) or Benactyzine or Brofaromine or Bupropion or Butriptylin* or Cianopramine or Cilobamine or Citalopram or (Chlorimipramin* or Clomipramin* or Chlomipramin* or Clorimipramine) or Clorgyline or Clovoxamine or (CX157 or Tyrima) or Dapoxetine or Deanol or Dibenzepin* or Demexiptilin* or Deprenyl or Desipramine or Desvenlafaxine or Dibenzepin or Dimetacrin* or (Dosulepin or Dothiepin) or Doxepin or Duloxetine or DVS-233 or Enilospirone or Eptapirone or Escitalopram or Etoperidone or Femoxetine or Fluotracen or Fluoxetine or Fluparoxan or Furazolidone or Fluvoxamine or Harmaline or Harmine or Hyperforin or Hypericum or John* Wort or Idazoxan or Imipramin* or Iprindole or Iproniazid* or Ipsapirone or Imipraminoxide or Isocarboxazid* or Lesopitron or Levomilnacipran or Lithium or Lofepramin* or (Lu AA21004 or Vortioxetine) or Lu AA24530 or LY2216684 or Maprotiline or Medifoxamine or Melitracen or Metapramine or Mianserin or Milnacipran or Minaprine or Mirtazapine or Moclobemide or Nefazodone or Nialamide or Nitroxazepine or Nomifensine or Norfenfluramine or Nortriptyline or Noxiptilin* or Opipramol or Oxaflozane or Paroxetine or Phenelzine or Pheniprazine or Pipofezin* or Pirandamine or Piribedil or Pirlindole or Pivagabine or Pizotyline or Propizepine or (Protriptylin* or Pertofrane) or Quinupramine or Quipazine or Reboxetine or Ritanserin or Rolipram or Scopolamine or Selegiline or Sertraline or (Setiptiline or Teciptiline) or Tandospirone or Tetrindole or Thiazesim or Thozalinone or Tianeptin* or Toloxatone or Tranylcypromine or Trazodone or Trimipramine or 5-Hydroxytryptophan or 5-HT or Tryptophan or Hydroxytryptophan or Venlafaxine or Viloxazine or Vilazodone or Viqualine or Zalospirone or Zimeldine or (Alaproclate or Caroxazone or Diclofensine or Fenfluramine)) .ti,ab,id,de.

64. or/56-63

[Mood Stabilisers or Antipsychotics]

65. MOOD STABILIZERS/

66. $\exp$ ANTICONVULSIVE DRUGS/

67. exp NEUROLEPTIC DRUGS/

68. ((mood stabili?er*1 or lithium or eslicarbazepine or licarbazepine or valnoctamide or carbamazepine or valproate or valproic acid or divalpro* or ziprasidone or gabapentin or lamotrigine or topiramate) or (antipsychotic*1 or amisulpride or aripiprazole or asenapine or cariprazine or clozapine or haloperidol or iloperidone or lurasidone or olanzapine or quetiapin* or paliperidone or prosulpride or risperidone)).ti,ab,id,de.

69. or/65-68

70. (9 and (55 or 64 or 69$)$ and 36)

71.37 or 68 (c3000 hits)

\section{OVID cross-search}

\section{MAINTENANCE THERAPY/}

2. (continuation or maintenance).ti.

3. ((continuation or maintenance) adj2 (efficacy or effectiveness or medicat* or pharmacotherap* or phase or study or therap* or psychotherap* or treatment*)).ti,ab,id,kf,kw.

4. ((continu* or maint*) adj (medicat* or pharmacotherap* or therap* or psychotherap* or treatment*)).ti,ab,id,kf,kw.

Continuation and maintenance treatments for persistent depressive disorder (Protocol)

Copyright (c) 2017 The Cochrane Collaboration. Published by John Wiley \& Sons, Ltd. 
5. or/1-4

6. chronic* depress*.ti,ab,id,kf,kw.

7. doubl* depress*.ti,ab,id,kf,kw.

8. DYSTHYMIC DISORDER/ or DYSTHYMIA/

9. dysthymi*.ti,ab,id,kf,kw.

10. MAJOR DEPRESSION/ or DEPRESSIVE DISORDER/ or DEPRESSIVE DISORDER, MAJOR/

11. "CHRONICITY (DISORDERS)"/ or CHRONIC ILLNESS/ or CHRONIC DISEASE/ or RECURRENCE/ or RECURRENT DISEASE/ or RECURRENCE RISK/ or REMISSION/

12. (10 and 11)

13. RECURRENT DEPRESSION/

14. (depress* adj2 recurr*).ti,ab,id,kf,kw.

15. persistent depressive disorder.ti,ab,id, $\mathrm{kf}, \mathrm{kw}$.

16. (6 or 7 or 8 or 9 or 12 or 13 or 14 or 15$)$

17. (5 and 16)

[De-duplicated $=1400]$

\section{Cochrane Central Register of Controlled Trials (CENTRAL)}

\#1 (continuation or maintenance):ti

\#2 ((continuation or maintenance) near/3 (efficacy or effectiveness or medicat* or pharmacotherap* or phase or study or therap* or psychotherap* or treatment $\left.\left.{ }^{*}\right)\right)$

\#3 ((continu* or maint*) next (medicat* or pharmacotherap* or therap* or psychotherap* or treatment*))

$\# 4$ (\#1 or \#2 or \#3)

\#5 “chronic* depress*”

\#6 "doubl* depress*”

\#7 MeSH descriptor: [DYSTHYMIC DISORDER] this term only

\#8 dysthymi*

\#9 MeSH descriptor: [DEPRESSIVE DISORDER] this term only

\#10 MeSH descriptor: [DEPRESSIVE DISORDER, MAJOR] this term only

\#11 MeSH descriptor: [CHRONIC DISEASE] explode all trees

\#12 MeSH descriptor: [RECURRENCER] this term only

$\# 13$ (\#9 or \#10) and (\#11 or \#12)

$\# 14$ (depress* near/3 recurr*)

$\# 15$ (persistent next depress*)

$\# 16$ (\#5 or \#6 or \#7 or \#8 or \#13 or \#14 or \#15)

$\# 17$ (\#4 and \#16)

\section{H I S T O R Y}

Protocol first published: Issue 11, 2017

\begin{tabular}{l|l|l}
\hline Date & Event & Description \\
\hline 17 June 2016 & Feedback has been incorporated & Feedback of editors incorporated \\
\hline
\end{tabular}

Continuation and maintenance treatments for persistent depressive disorder (Protocol)

Copyright $(2017$ The Cochrane Collaboration. Published by John Wiley \& Sons, Ltd. 


\section{CONTRIBUTIONSOFAUTHORS}

SL, KM, AJ, RM, BW, MH, and LK developed the protocol.

\section{DECLARATIONSOF INTEREST}

Alessa Jansen, Levente Kriston, and Martin Härter coordinated the update of the S3 Guideline/National Clinical Practice Guideline "Unipolar Depression" (DGPPN 2015). The expert association DGPPN (editor of the S3 Guideline) provided financial support for the preparation of the Guideline Update to the coordinators' institution (Department of Medical Psychology, University Medical Center Hamburg-Eppendorf).

Katja Machmutow, Birgit Watzke, Alessa Jansen, Levente Kriston, Ramona Meister, Martin Härter, and Sarah Liebherz report participating in publicly funded investigator-initiated primary studies and systematic reviews of interventions for patients with depression.

Katja Machmutow, Birgit Watzke, Alessa Jansen, Ramona Meister, Martin Härter and Sarah Liebherz have a formal training in behavioral psychotherapy.

\section{SOURCES OF SUPPORT}

\section{Internal sources}

- No sources of support supplied

\section{External sources}

- German Ministry of Education and Research, Germany. grant $01 \mathrm{KG} 1403$ 